$\overline{\text { Note }}$

\title{
Analysis of Red Cabbage Colors in Commercial Foods Using High Performance Liquid Chromatography with Photodiode Array Detection-Mass Spectrophotometry
}

(Received August 24, 1996)

\author{
Takashige ShImizu*1, Teruyo MuroI*1, Takahito ICHI*1, \\ Mikio NaKamura*1 and Kunitoshi Yoshihira*2 \\ (*1San-Ei Gen F. F. I., Inc.: 1-1-11, Sanwa-cho, Toyonaka-city, Osaka 561, Japan; \\ ${ }^{* 2}$ Graduate School at Toa University: 2-1 Ichinomiya gakuen-cho, \\ Shimonoseki-city, Yamaguchi 751, Japan)
}

\begin{abstract}
A method for analysis of red cabbage colors in processed foods has been developed using high performance liquid chromatography with photodiode array detection-mass spectrophotometry (PDA-LC-MS). The beverages were simply diluted with water. The red cabbage colors were extracted from the jellies with hot water, cleaned up using a C18 cartridge and injected into the PDA-LC-MS system to carry out determination and identification simultaneously. The recoveries of red cabbage colors from laboratory-prepared beverage and jelly were 90.0 and $55.5 \%$, respectively. The detection limit of the total colors was $5 \mu \mathrm{g} / \mathrm{g}$ calculated as cyanidin 3-glucoside. Processed beverage and jelly were analyzed by this method, and the red cabbage colors in the samples were identified and determined successfully.
\end{abstract}

Key words: red cabbage, Brassica oleracea L. var. Capitata DC.; anthocyanin; high performance liquid chromatography with photodiode array detection-mass spectrometry (PDA-LC-MS); food color

\section{Introduction}

Red cabbage colors are anthocyanin group compounds extracted from red cabbage (Brassica oleracea L. var. Capitata DC.) and they are widely used in beverages, jellies, and candies. It is known that the red cabbage contains many pigments and the structures of 7 major pigments have been elucidated by Nakatani et $a l{ }^{11.2)}$ and Baublis et al. ${ }^{3)}$ As shown in Fig. 1, cyanidin 3-glucoside is the basic structure of the pigments and they differ from one another in the structures of $R_{1}$ and $R_{2}$.

In 1995, the Japanese Food Sanitation Law was revised to regulate the usage of natural colorants to the same level as synthetic food colorants. Accordingly, it has became necessary to develop reliable analysis methods for various natural colorant materials in foods. However. such an analysis method has not yet been reported for the red cabbage colors. In addition, a standard of red cabbage color is not commercially available. We previously established an analysis method for gardenia yellow colors used in processed food ${ }^{4}$. In this method, photodiode array (PDA) detection HPLC and electrospray ionization (ESI) liquid chromatography-mass spectrometry (LC-MS) were used to determine and identify the colors of gardenia yellow.

A combination of PDA-HPLC and LC-MS (PDA-LC-MS system) is advantageous for simple and reliable analysis, because determination and identification can be accomplished at the same time. Therefore, we sought to apply the system to the analysis of red cabbage colors. This paper describes the techniques for the preparation of a working standard from red cabbage leaves and for the quantitative and 
qualitative analysis of red cabbage colors in processed food using the PAD-LC-MS system.

\section{Materials and Methods}

\section{Apparatus}

The HPLC system consisted of Jasco PU-970 pumps, a Jasco AS-950 autoinjector, a Jasco MD910 photodiode array detector (250 to $600 \mathrm{~nm}$ ) and a VG Biotech Platform mass spectrophotometer. An analytical column, Nucleosil $5 \mathrm{C} 18$ ( $\phi 4.6 \mathrm{~mm} \times 250 \mathrm{~mm}$, Chemco) was placed between the autoinjector and detectors. A Sep-Pak $\mathrm{tC}_{18}$ cartridge (Waters) was examined for absorption of pigments and clean-up of extracts from beverages and jellies. UV-Vis spectra were recorded on a Hitachi U-3200.

\section{Reagent}

Hydrochloric acid $(\mathrm{HCl})$, trifluoroacetic acid (TFA), acetic acid and formic acid were of analytical grade from Wako. Methanol, ethanol, and tetrahydrofuran (THF) were of HPLC grade from Wako. Sucrose was of guaranteed grade from Kishida. Water was distilled and deionized. Amberlite XAD-2000 for column chromatography (C.C) and Sephadex LH-20 for C.C were purchased from Organo and Pharmacia Fine Chemicals, respectively. Grape flavor and citric acid of food additive grade, grape juice (anthocyanin free) and gelatin of food grade were supplied through San-Ei Gen F.F.I.

\section{Samples}

Five beverages and five jellies were purchased in the markets of Osaka within 3 days of manufacturing. Red cabbage (Brassica oleracea $\mathrm{L}$. var. capitata DC.) cultivated in Okayama, Japan was used for making a Standard Red Cabbage Color (SRCC).

\section{Preparation of standard red cabbage color (SRCC)}

One $\mathrm{kg}$ of red cabbage was cut into small pieces and steeped in 5\% acetic acid aqueous solution $(10 \mathrm{~L})$ at $25^{\circ} \mathrm{C}$ overnight, then filtered with filter paper (No. 5C, Advantec Toyo). The filtrate was charged on an Amberlite XAD-2000 column $(\phi 45 \mathrm{~mm} \times 500 \mathrm{~mm})$ and washed with $1 \mathrm{~L}$ of water. The absorbed pigments were eluted with $500 \mathrm{~mL}$ of $70 \%$ ethanol solution. The eluate was evaporated to dryness in vacuo at below $40^{\circ} \mathrm{C}$. The residue was dissolved in a small amount of $80 \%$ ethanol solution and applied to a Sephadex LH-20 column $(\phi 30 \mathrm{~mm} \times$ $500 \mathrm{~mm}$ ) using $80 \%$ ethanol as the solvent. The reddish fractions were collected and evaporated to dryness in vacuo at below $40^{\circ} \mathrm{C}$. The residue was dried in a desiccator in vacuo at $40^{\circ} \mathrm{C}$ for 48 $\mathrm{h}$ and powdered $(850 \mathrm{mg})$. This pigment was used in the following experiments as standard red cabbage color (SRCC).

\section{Total color concentration of SRCC}

One mg of the SRCC was dissolved in a small amount of $0.1 \mathrm{~mol} / \mathrm{L} \mathrm{HCl}$ aqueous solution, and made up to exactly $100 \mathrm{~mL}$ with $0.1 \mathrm{~mol} / \mathrm{L} \mathrm{HCl}$ aqueous solution. The absorbance of this solution was measured at $530 \mathrm{~nm}$ with $0.1 \mathrm{~mol} / \mathrm{L}$ $\mathrm{HCl}$ aqueous solution as a reference. The concentration of SRCC was calculated as cyanidin 3-glucoside $\left(E_{1}^{1 \% \mathrm{~cm}}=257.4\right)^{5)}$.

\section{Calibration curve}

A $20 \mathrm{mg}$ portion of the SRCC was dissolved in a small amount of water, and made up to exactly $200 \mathrm{~mL}$ with water as the test solution. Then $75,50,25,10,5$, and $0 \mathrm{~mL}$ portions of test solution were each diluted with water. Each solution was injected into the PDA-LC-MS system. A calibration curve was made from the total peak areas of seven pigments in red cabbage color and each concentration using an absolute calibration curve method.

\section{Preparation of sample solution}

1) Beverage

Ten $\mathbf{g}$ of processed beverage was weighed accurately, diluted with $70 \mathrm{~mL}$ of water, if necessary filtered with filter paper (No. 5C), and applied to the Sep-Pak $\mathrm{tC}_{18}$ cartridge. The cartridge was washed with $10 \mathrm{~mL}$ of water, and then eluted with $2 \mathrm{~mL}$ of $80 \%$ ethanol solution containing $0.5 \%$ TFA. The eluate was evaporated to dryness in vacuo at below $40^{\circ} \mathrm{C}$. The residue was dissolved and made up to $10 \mathrm{~mL}$ with water. This solution was used as the test solution for PDA-LC-MS.

2) Jelly

Ten $g$ of each processed jelly was weighed accurately and extracted with $70 \mathrm{~mL}$ of water at $60^{\circ} \mathrm{C}$ by stirring for $30 \mathrm{~min}$. The solution was 


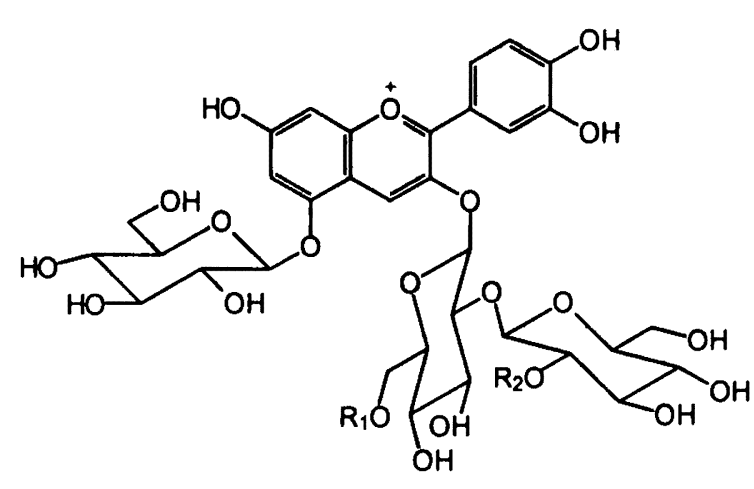<smiles>CC(=O)/C=C/c1ccc(O)cc1</smiles><smiles>COc1cc(/C=C/C(C)=O)ccc1O</smiles><smiles>COc1cc(/C=C/C(C)=O)cc(OC)c1O</smiles>

p-coumaryl

\begin{tabular}{cccr}
\hline \hline Peak & $\mathrm{R}_{\mathbf{l}}$ & $\mathrm{R}_{\mathbf{2}}$ & $\begin{array}{c}\text { Content } \\
(\%)\end{array}$ \\
\hline A & H & Sinapyl & 7.2 \\
B & Sinapyl & $H$ & 10.3 \\
C & Ferulyl & $H$ & 6.0 \\
D & p-Coumaryl & H & 7.9 \\
E & Sinapyl & Sinapyl & 30.5 \\
F & Ferulyl & Sinapyl & 11.6 \\
G & $p$-Coumaryl & Sinapyl & 8.6 \\
\hline
\end{tabular}

Fig. 1. Structures and contents of pigments in red cabbage colors

filtered through filter paper (No. 5C). The filtered solution was treated in the same manner as described in the case of the beverages.

\section{Recovery test}

The beverage and jelly for the recovery test of red cabbage color were prepared in the laboratory.

\section{1) Beverage}

Twelve $\mathrm{g}$ of sugar, $0.2 \mathrm{~g}$ of citric acid, $0.1 \mathrm{~g}$ of grape flavor and $3.5 \mathrm{mg}$ of SRCC $(30 \mu \mathrm{g} / \mathrm{g}$ as cyanidin 3-glucoside) were dissolved in $87.7 \mathrm{~g}$ of water, placed in a bottle and sterilized at $80^{\circ} \mathrm{C}$ for $10 \mathrm{~min}$.

\section{2) Jelly}

Twenty-five $g$ of sugar and $1 \mathrm{~g}$ of gelatin were added to $63.8 \mathrm{~g}$ of water, and dissolved with heating. After the solution had cooled to about $60^{\circ} \mathrm{C}, 0.2 \mathrm{~g}$ of citric acid, $10 \mathrm{~g}$ of grape juice (anthocyanin free), and $5.7 \mathrm{mg}$ of SRCC $(50 \mu \mathrm{g} / \mathrm{g}$ as cyanidin 3-glucoside) were added and mixed. This solution was placed in a container and sterilized at $80^{\circ} \mathrm{C}$ for $10 \mathrm{~min}$.

\section{HPLC conditions}

Analytical HPLC was performed on a Nucleosil $5 \mathrm{C} 18$ column $(\phi 4.6 \mathrm{~mm} \times 250 \mathrm{~mm})$ at $30^{\circ} \mathrm{C}$ using a gradient formed from solvent A (10\% formic acid) and solvent B (methanol-THF (1 : 1)) at a flow rate of $1 \mathrm{~mL} / \mathrm{min}$. The pigments were analyzed under the following gradient conditions: $0-15 \mathrm{~min}$, a linear gradient from $5 \%$ to $10 \%$ solvent $B$ in solvent $A ; 15-50$ min, isocratic elution with $10 \%$ solvent $B$ in solvent $A$ with detection using PDA monitored at $530 \mathrm{~nm}$ and the mass spectrophotometer (MS). The injection volume was $100 \mu \mathrm{L}$. The flow rates of PDAHPLC and LC-MS were $0.95 \mathrm{~mL} / \mathrm{min}$ and 0.05 $\mathrm{mL} / \mathrm{min}$, respectively, via a splitter. 
Table 1. Protonated Molecules and Fragment Ions of Red Cabbage Colors

\begin{tabular}{cccc}
\hline \hline Pigment & \multicolumn{3}{c}{$\begin{array}{c}\text { Prominent ions } \\
\text { (Relative intensity) }\end{array}$} \\
\hline A & $979[\mathrm{M}+\mathrm{H}]^{+},(80)$ & $449[\mathrm{Cy}+\mathrm{Glc}]^{+},(100)$ & $287[\mathrm{Cy}]^{+},(100)$ \\
B & $979[\mathrm{M}+\mathrm{H}]^{+},(95)$ & $449[\mathrm{Cy}+\mathrm{Glc}]^{+},(100)$ & $287[\mathrm{Cy}]^{+},(100)$ \\
C & $949[\mathrm{M}+\mathrm{H}]^{+},(48)$ & $449[\mathrm{Cy}+\mathrm{Glc}]^{+},(90)$ & $287[\mathrm{Cy}]^{+},(30)$ \\
D & $919[\mathrm{M}+\mathrm{H}]^{+},(50)$ & $449[\mathrm{Cy}+\mathrm{Glc}]^{+},(80)$ & $287[\mathrm{Cy}]^{+},(60)$ \\
E & $1,185[\mathrm{M}+\mathrm{H}]^{+},(100)$ & $449[\mathrm{Cy}+\mathrm{Glc}]^{+},(100)$ & $287[\mathrm{Cy}]^{+},(100)$ \\
F & $1,155[\mathrm{M}+\mathrm{H}]^{+},(60)$ & $449[\mathrm{Cy}+\mathrm{Glc}]^{+},(70)$ & $287[\mathrm{Cy}]^{+},(25)$ \\
G & $1,125[\mathrm{M}+\mathrm{H}]^{+},(48)$ & $449[\mathrm{Cy}+\mathrm{Glc}]^{+},(50)$ & $287[\mathrm{Cy}]^{+},(30)$ \\
\hline
\end{tabular}

M: Molecule; Cy: Cyanidin; Glc: Glucose

Table 2. Maximum Absorption Wavelength of Red Cabbage Colors

\begin{tabular}{cccc}
\hline \hline Pigment & \multicolumn{3}{c}{$\lambda_{\max }(\mathrm{nm})^{\mathrm{a})}$} \\
\hline A & 531 & 332 & 283 \\
B & 527 & 331 & 280 \\
C & 527 & 327 & 280 \\
D & 527 & 319 & 280 \\
E & 539 & 332 & 229 \\
F & 539 & 331 & 295 \\
G & 539 & 332 & 229 \\
\hline
\end{tabular}

a) Measured by PDA-HPLC

\section{MS conditions}

MS conditions were as follows; ion source, electrospray ionization (ESI); mode, positive; ionization conditions, ion source temperature, $70^{\circ} \mathrm{C}$; nebulizer gas, nitrogen gas; gas flow, $10 \mathrm{~L} / \mathrm{h}$; skimmer voltage, $100 \mathrm{~V}$.

\section{Qualitative and quantitative analysis}

The seven colors of red cabbage were identified from the retention times at $530 \mathrm{~nm}$ by PDAHPLC, the protonated molecules in the mass spectra and the maximum absorption wavelengths in the visible spectra obtained with the PDA-LC-MS system. The concentration of red cabbage colors was calculated from the total areas of the seven peaks in the chromatogram at the wavelength of $530 \mathrm{~nm}$ using the calibration curve obtained by PDA-HPLC.

\section{Results and Discussion}

The total color concentration and composition of SRCC

Total color concentration of SRCC was measured with a spectrophotometer at $530 \mathrm{~nm}$. The absorbance of SRCC solution at the concentration of $10 \mu \mathrm{g} / \mathrm{mL}$ in $0.1 \mathrm{~mol} / \mathrm{L} \mathrm{HCl}$ aqueous solu.

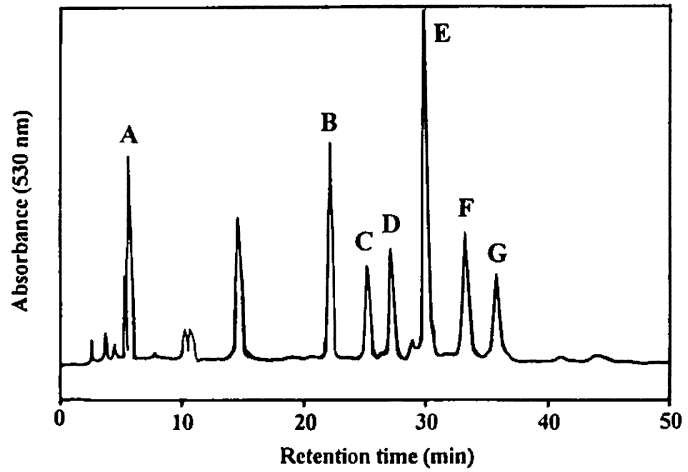

Fig. 2. HPLC chromatogram of red cabbage colors

Column: Nucleosil $5 \mathrm{C}_{18}(\phi 4.6 \mathrm{~mm} \times 250 \mathrm{~mm})$; flow rate: $1.0 \mathrm{~mL} / \mathrm{min}$

Detector: photodiode array $(530 \mathrm{~nm})$

Mobile phase: solvent A (10\% formic acid), solvent $B$ (methanol-THF =1:1)

Gradient rate: $0-15 \mathrm{~min}$ (95-90\% solvent

A; linear gradient $) \rightarrow 15-50$ min $(90 \%$ solvent A hold)

tion was 0.224 . From this result, the concentration of SRCC was estimated to be $87.0 \%$ as cyanidin 3-glucoside $\left(\left.E\right|_{\mathrm{cm}} ^{\%}=257.4\right)^{5)}$, which is the basic structure of the seven pigments in red cabbage color.

The pigments of red cabbage colors were analyzed using the PDA-LC-MS system. Figure 1 and Tables 1 and 2 show the HPLC profile at 530 $\mathrm{nm}$, the protonated molecule and the maximum absorption wavelengths of each color as determined by the PDA-LC-MS system. Figure 2 showed that red cabbage colors in clude seven colors (labeled A, B, C, D, E, F and G in order of elution) with a composition of $\mathrm{A}: 7.2 \%$, B: $10.3 \%$, C: $6.0 \%$, D: $7.9 \%$, E: $30.5 \%, \mathrm{~F}: 11.6 \%$, and G: $8.6 \%$. As shown in Table 2, prom- 
inent fragment ions were observed at $\mathrm{m} / \boldsymbol{z}$ 449 and 287 for all pigments, corresponding to $\left[\mathrm{C}_{21} \mathrm{H}_{21} \mathrm{O}_{11}\right]^{+}(\mathrm{Cy} 3-\mathrm{G})$ and $\left[\mathrm{C}_{15} \mathrm{H}_{12} \mathrm{O}_{6}\right]^{+}(\mathrm{Cy})$, respectively. This indicates that these ions originate from glucosyl cyanidin structure in the pigments. From the mass data described above and those of authentic materials, the seven pigments of SRCC were identified as shown in Fig. 1.

\section{Calibration curve and detection limit}

The calibration curve of SRCC using PDAHPLC showed high linearity between 0 to 75 $\mu \mathrm{g} / \mathrm{g}$. The detection limit of red cabbage colors was $5 \mu \mathrm{g} / \mathrm{g}$, calculated as cyanidin 3 -glucoside.

\section{Recovery test}

The recoveries of SRCC from laboratoryprepared beverage and jelly using PDA-HPLC were 90.0 (average of five duplicates) and $55.5 \%$ (average of five duplicates), respectively. The composition of pigments recovered in this examination was very similar to that in SRCC. Therefore, the red cabbage colors are very stable in the laboratory sample, in spite of heating.

\section{Application to products on the market}

In order to confirm the applicability of this PDA-LC-MS method, processed beverages and jellies were analyzed. In the chromatograms of samples, peaks of colors were observed at the same retention times as the 7 colors of SRCC.

In addition, the colors in the sample showed the same mass and UV-Vis spectra as those of SRCC, and the composition of colors in the sample was closely similar to that of SRCC, indicating that the sample contained the same colors as SRCC. These results suggest that the PDA-LC-MS is applicable to identify the red cabbage colors in processed food samples. In order to determine the actual concentration of red cabbage colors in the samples, HPLC quantitative data were corrected for the recovery of each sample ( 90.0 and $55.5 \%$ ), and values of 26.5 and $33.4 \mu \mathrm{g} / \mathrm{g}$ were obtained as average (five determinations) concentrations of cyanidin 3glucoside in the processed beverages and jellies, respectively. The coefficients of variations were 14.3 and $11.2 \%$, respectively. These values are considered reasonable in terms of the usual amounts of red cabbage colors added to these foods.

The low recovery of SRCC from jelly was considered to be a consequence of tight binding to the gelatin matrix, which has a triple helix structure after gelling ${ }^{6}$. In this respect, further studies are necessary.

\section{Conclusion}

A method for the analysis of red cabbage colors in beverage and jellies has been established using a PDA-LC-MS system. This is very effective to analyze the pigments simply and reliably, because the identification and determination of the pigments can be carried out simultaneously. The recoveries of red cabbage colors from laboratory-prepared beverage and jelly were 90.0 and $55.5 \%$, respectively. The detection limit of total pigments was $5 \mu \mathrm{g} / \mathrm{g}$, calculated as cyanidin 3-glucoside. The recovery from jelly is low, but the present method is considered to be applicable to the quantitative and qualitative analysis of red cabbage colors in commercial foods.

\section{References}

1) Nakatani, N., Ikeda, K., Nakamura, M., Kikuzaki, H.: Chemistry Express 2, 555-558 (1987).

2) Ikeda, K., Kikuzaki, H., Nakatani, N.: ibid. 2, 563566 (1987).

3) Baublis, A., Spomer, A., Berber-Jimenez, M. D.: J. Food Sci. 59, 1,219-1,223 (1994).

4) Ichi, T., Higashimura, Y., Katayama, T., Koda, T., Tada, M.: J. Food Hyg. Soc. Japan 36, 482-489 (1995).

5) Pericles, M. ed.: "Anthocyanins as Food Colors" p. 181-207 (1982) Academic Press, New York.

6) Kobayashi, T.: Foods Food Ingredients J. Jpn. No. $170,82-88$ (1996). 